\section{AVANZANDO EN EL DIÁLOGO DE SABERES}

\author{
Sylvia Contreras Salinas \\ Doctoranda del programa "Pedagogía de la Diversidad Socio- \\ cultural". Departamento de Teoría e Historia de la Educación. \\ Universidad Complutense de Madrid. \\ Becaria Conicyt del Gobierno de Chile. \\ nemesis.syl@gmail.com
}

Mónica Ramírez Pavelic Doctoranda del Programa de Doctorado: Desarrollo psicológico, aprendizaje y educación: Perspectivas contemporáneas. Universidad Autónoma de Madrid. trapecio@gmail.com

Cómo citar este artículo/ Citation: Contreras Salinas, S. y Ramírez Pavelic, M. (2013). Avanzando en el diálogo de saberes. Arbor, 189(759):a011. doi: http://dx.doi.org/10.3989/ arbor.2013.759n1010

Recibido: 31 agosto 2009; Aceptado: 18 marzo 2011.

RESUMEN: Este trabajo intenta avanzar en el debate sobre la ceguera en la construcción del conocimiento, originada por la incapacidad de visualizar otras formas de vida que se desarrollan fuera del sistema hegemónico, marcado esencialmente por la ideología capitalista y eurocéntrica. Para esta empresa, optamos por presentar a modo de ejemplo una serie de enunciados que mostrarán discursos marcados por prácticas y formas de vida de habitantes de sectores rurales del Centro y Sur de Chile. El procedimiento utilizado consistió en visibilizar nuestras propias concepciones paradigmáticas para "ver" en todo el sentido de la palabra, discursos diversos; todo esto desde una perspectiva hermenéutica. Entre las conclusiones destaca la idea del "andar", la paciencia y las recurrentes metáforas construidas desde la relación con la naturaleza.

PALABRAS CLAVE: Diálogo de saberes; ruralidad; patrimonio cultural inmaterial.

\section{ADVANCING IN THE DIALOGUE OF KNOWLEDGE}

Copyright: (c) 2013 CSIC. Este es un artículo de acceso abierto distribuido bajo los términos de la licencia Creative Commons Attribution-Non Commercial (by-nc) Spain 3.0.

ABSTRACT: This paper attempts to advance the debate on the blindness in the construction of knowledge, caused by the inability to see other life forms that develop outside of the hegemonic system, marked mainly by the capitalist and Eurocentric ideology. For this undertaking, we chose to present as examples a series of statements that show speeches marked by practices and lifestyles of people in rural areas of central and southern Chile.

The procedure used was to make our own paradigmatic conceptions visible so as to "see" -in every sense of the word- diverse discourses ; all from a hermeneutic perspective. Among the findings highlighted the idea of "gait", patience and recurring metaphors constructed from the relationship with nature.

KEYWORDS: Dialogue of knowledge; rurality; intangible cultural heritage. 
"El ser viviente no puede sobrevivir en un entorno más que con y por un conocimiento de ese contorno. Sin conocimiento, la vida no es ni viable ni vivible" (Morín, 1988, 220)

\section{INTRODUCCIÓN}

En la actualidad nos vemos constantemente bombardeados por discursos pesimistas sobre nuestro presente y futuro, estos transitan desde la concepción del individuo como un sujeto no-social, al reconocimiento de un sujeto que re-nace continuamente, definiendo la memoria y las tradiciones como cargas que obstaculizan sus proyectos. (Bauman, 2003 y 2005; Castells, Giddens, Touraine, 2002; Beck 1998). Desde estos supuestos, se nos presenta la realidad como una sola; sabemos, sin embargo, que este fenómeno surge de una enfermedad moderna: "la hiper-simplificación que ciega la complejidad de lo real" (Morín, 1990, 34). Los efectos de estas teorías implican la no creencia en otras formas de conocimiento, esto a su vez permite que la realidad sea encubierta por ideas generalmente dogmáticas que no hacen más que petrificar las relaciones entre conocimiento y experiencia.

En este contexto, el presente trabajo pretende ser una humilde invitación a soltar nuestras amarras cognitivas y epistémicas, para entregarnos a la posibilidad de dialogar con otras formas de conocer las múltiples realidades que nos configuran, en pos de construir relaciones y tramas que sustenten al sujeto social. En otras palabras, avanzar en la "solidaridad" como un "conocimiento-re-conocimiento" (De Sousa Santos, 2003), elevando al otro/a a la categoría de sujeto.

Esta invitación busca además del examen de la propia realidad social (para traducirla en investigación sistemática y docente) transmitir nuestra explícita intención de transformar esa realidad en co-participación con otros/as.

Se espera alcanzar este propósito, a través de una revisión del estado del arte del tema y de la exposición de pensamientos que construyen habitantes de sectores rurales, bajo un enfoque que tiene como puntos de apoyo la experiencia personal y narrativa de nuestros/as informantes, adoptando una perspectiva metodológica cualitativa en la cual se resalta la singularidad y profundidad de los relatos de cada uno/a de ellos/as.

\section{ESTADO DE LA CUESTIÓN}

Parece ser como si de pronto "nuestra razón, que nos parecía el modo de conocimiento más seguro, descubre en sí una mancha ciega. ¿Qué es nuestra razón? ¿Es universal? ¿Racional? ¿No puede transmutarse en su contrario sin darse cuenta de ello? ¿No empezamos a descubrir que hemos ignorado, despre- ciado, destruido tesoros de conocimiento en nombre de la lucha contra la ignorancia?" (Morin, 1988, 18).

La respuesta a estas interrogantes puede venir de la reflexión epistemológica, una reflexión que se hace con otros/as y donde se busca construir comprensiones que expliciten los límites y ambigüedades de las ideas que sustentan las ciencias. No podemos olvidar que "las creencias son, en efecto, una producción histórica construidas por humanos y para humanos; contienen posibilidades de liberación, una gran belleza estética, una notable fiabilidad, pero pueden también convertirse en mitos, ser origen de destrucción y factor de desigualdad social" (Fourez, 2008, 10). La iniciativa adopta entonces un carácter imperativo: el dialogar con los/as otros/as que por demasiado tiempo hemos tratado de acallar, este ejercicio posibilitará que ellos/as puedan facilitarnos desde sus saberes nuevas posibilidades de re-construir nuestros y sus conocimientos.

Esta demanda se hace especialmente patente en el contexto de la pobreza rural y el deterioro ambiental. Al respecto, múltiples organizaciones, en su mayoría alternativas, denuncian que a menos de cincuenta años de los inicios de la Revolución Verde, existe un mundo rural cada vez menos diverso, una agricultura cada vez más homogénea y concentrada, manteniéndose en ellos la pobreza y haciendo en ocasiones más grande la brecha entre los sectores rurales y urbanos. Se aprecia además, que los cultivos están fuertemente controlados por el comercio internacional a través de las grandes corporaciones, ocasionando que la producción campesina se haya estancado, sobre todo porque los campesinos tienen cada vez menos tierra para sembrar, además del efecto inmediato de la deforestación, que no sólo ha significado deterioro ambiental, sino importantes pérdidas de fuentes de alimentación humana y animal (Grain, 2009).

Por su parte la FAO y la UNESCO ${ }^{1}$ entregan datos alarmantes, entre ellos: que existen 840 millones de personas mal nutridas; 1.500 millones de personas que no tienen acceso al agua potable, de las cuales la mayoría vive en zonas rurales. Coincidentemente cerca del $70 \%$ de los pobres del mundo viven en las zonas rurales y se prevé que para el 2025 el $60 \%$ de la población pobre seguirá viviendo en estas zonas.

En este marco, la FAO y la UNESCO ${ }^{2}$ lanzan en el año 2002 un programa en el marco de la iniciativa de Educación para todos (EPT), focalizado en la atención de la población rural. Al parecer, este programa se funda en la noción de que el conocimiento constituye un elemento clave para fortalecer a las comunidades rurales y asegurar un desarrollo global sustentable.

Así, el Programa 21 (programa de las Naciones Unidas para promover el desarrollo sostenible) consti- 
tuye "un plan de acción exhaustivo que habrá de ser adoptado universal, nacional y localmente por organizaciones del Sistema de Naciones Unidas, Gobiernos y Grupos Principales de cada zona en la cual el ser humano influya en el medio ambiente" ${ }^{\prime 3}$.

Esta iniciativa hace alusión específicamente al concepto de "patrimonio cultural inmaterial". En el texto de La Convención 2003, se define el PCI (Patrimonio cultural inmaterial), como "los usos, representaciones, expresiones, conocimientos y técnicas que las comunidades, los grupos y, en algunos casos, los individuos reconocen como parte integrante de su patrimonio cultural" ${ }^{\prime 4}$.

En esta línea la convención reafirma la importancia y necesidad de conservar este patrimonio, considerado el eje de la diversidad cultural, el cual se manifiesta en particular en los siguientes ámbitos:

- tradiciones y expresiones orales, incluido el idioma como vehículo del patrimonio cultural inmaterial;

- artes del espectáculo;

- usos sociales, rituales y actos festivos;

- conocimientos y usos relacionados con la naturaleza y el universo;

- técnicas artesanales tradicionales ${ }^{5}$.

Asimismo, apreciamos que se encaminan algunas medidas para garantizar la viabilidad del patrimonio cultural inmaterial: "la identificación, documentación, investigación, preservación, protección, promoción, valorización, transmisión -básicamente a través de la enseñanza formal y no formal- y revitalización de este patrimonio en sus distintos aspectos" ${ }^{\prime \prime}$.

En este marco, los saberes tradicionales penetran con mayor fuerza, a pesar de las múltiples acciones emprendidas para eliminarlos. Estos saberes reciben distintas denominaciones, entre ellas:

"saberes locales", "sabiduría popular", "folklore", "ciencia indígena", "ciencias nativas", "conocimiento popular", "ciencia del pueblo, "conocimiento campesino", "saberes subyugados", "tradición científica no occidental", "ciencia emergente" y en la literatura anglosajona se les denomina también "tradicional knowledge, non western knowledge o traditional ecological knowledge" (Leff, 2002, 500).

Sin embargo, debemos considerar que estas formas de apropiación cognitiva sólo son posibles si los/as habitantes de los sectores rurales se plantean como necesidad fortalecerlas dentro de su patrimonio cultural y de su cosmovisión de los sistemas de saberes, formas simbólicas de percepción, significación y relación con la naturaleza, además de sus normas culturales y de organización social para la gestión de un desarrollo propio.

Creemos que esto debe hacerse mediante una estrategia que se aleje de "la acción extensionista que implica cualquiera que sea el sector en que se realice, la necesidad que sienten aquellos que llegan hasta "la otra parte del mundo", de considerarla inferior, para, a su manera, normalizarla. Para hacerla más o menos, semejante a su mundo" (Freire, 1998, 21).

Consideramos que la gestión del conocimiento que busca salvaguardar el patrimonio cultural inmaterial, debería iniciarse con la toma de conciencia. "La consciencia es inseparable del pensamiento, que es inseparable del lenguaje. La consciencia es la emergencia del pensamiento reflexivo del sujeto sobre sí mismo, sobre sus operaciones, sobre sus acciones" (Morín, $1988,134)$. Lo que se espera entonces, es que cada sujeto participe en la producción del conocimiento.

Por su parte, Grain nos informa sobre:

"una característica especialmente esperanzadora: la reactivación de los sistemas campesinos de construcción de saberes, sistemas que fusionan formas colectivas y personales de observación, experimentación e intercambio, y que al saber unen el respeto, la espiritualidad y un conjunto de normas sociales localmente definidas. Esta búsqueda permite la generación y reactivación autónoma de saberes por parte de comunidades y familias y, a fin de cuentas, el florecimiento, de nuevo, de la creatividad social más antigua de la humanidad"7.

Como contrapunto a esta eminente realidad, se apela a que los profesionales asuman un compromiso social, pues aquí definitivamente la neutralidad no existe.

Desde esta perspectiva creemos que: "tener un compromiso social, es no solo una forma apropiada para reconstruir la sociedad, sino también un reto para crear una ciencia seria que sea propia a la vez. Ésta es aquella disciplina que, al enfocar las necesidades y objetivos supremos de la sociedad local, llena también todos los requisitos académicos de acumulación del conocimiento, la formación de conceptos y la sistematización universal" (Fals Borda, 1973, 95).

\section{MÉTOdO}

El presente trabajo, se enmarca en el contexto de la potencialidad de los relatos, narraciones basadas en la confianza de que se pueden comprender realidades por medio de la interpretación de esos textos; generándose una relación dialéctica entre lo que expresa el texto y la subjetividad del que lo interpreta, una relación que tiene como resultado final "el diálogo". 
No pretendemos en este trabajo construir un diálogo, pues no se dan las condiciones de una real comunicación, sin embargo, sí podemos mostrar un mundo a través de la mirada de otros/as que generalmente no tiene una tribuna en la esfera de la producción de conocimientos.

Más que una interpretación de los datos que entregan los relatos, buscamos descubrir otros saberes, desde nuestra limitada capacidad para escapar de los paradigmas imperantes y de las formas naturalizadas de conceptualizar la realidad. En ese sentido, este trabajo requirió un gran esfuerzo personal, el de dejar atrás las pretensiones de imponer nuestra propia interpretación, priorizando el respeto por otras formas de cognición, distintas a las que normalmente estamos acostumbrados, pero igualmente legítimas y dignas de atender y admirar.

Importante mencionar que el estudio se realizó en base a entrevistas narrativas grabadas y posteriormente transcritas. Se revisaron un total de 18 entrevistas, realizadas entre el 2007-2008 (10 mujeres y 8 hombres de sectores rurales de la zona centro y sur de Chile).

El procedimiento realizado consistió en las lecturas y relecturas de transcripciones, hasta descubrir, en la medida de lo posible, lo que nos parecía una idea, pensamiento o conceptualización factible de asociar con un tipo de saber local y/o campesino.

La actitud de enfrentamiento a las narraciones fue teñida por la apertura a los cuestionamientos que se originaron en la construcción de las formulaciones teóricas y conceptuales que anteceden a este apartado.

En este ejercicio se ha dado más bien una búsqueda introspectiva, produciéndose un conocimiento autoreflexivo que explicita los sentidos subjetivos que vamos reconociendo en lo que somos y hacemos, y en especial en lo que son y hacen los otros/as.

Este enfoque nos ofreció la posibilidad de acercar la imagen del individuo, que generalmente vemos como algo abstracto (hablamos de los/as pobres, los/ as campesinos/as, las mujeres, etc.) a algo más concreto, singular y complejo: es decir, un acercamiento a otras formas de vivir y de ser.

El criterio de validez de esta investigación tendríamos que "buscarlo en ciertos rasgos del texto que nace de la investigación: en la coherencia, el consenso, sobre todo en su utilidad para hacernos ver lo que de otro modo quedaría probablemente oculto" (Gil \& Jover, 2000, 114).

Por lo tanto, los/as lectores/as se encontrarán con citas, que a nuestro modo de ver, muestran ideas, conceptos y/o construcciones teóricas de algunos habitantes de sectores rurales y podrán darse cuenta, al igual que nosotras, que esas construcciones, ya están formalizadas y generalizadas, y por tanto no requieren la mediación de "científicos/as". Es por esto que optamos por no hacer segundas construcciones, mostrando solo construcciones de primer grado (Flick, 2004).

Apreciamos que estos significados son construidos dentro de redes de relaciones, por lo que es imposible desligarlos de su contexto textual o fijar unas relaciones determinadas para su uso en contextos futuros (Woolgar, 1988 citado en Cubero, 2005).

\section{Ciertas ReConstrucciones de SABERES}

Las narraciones dan cuenta de experiencias que otorgan significado a recuperar el saber del sujeto que los narra, constituyéndose en relatos llenos de pensamientos que estructuran vivencias e interacciones, donde se evidencia además un principio epistemológico.

La reconstrucción que presentamos se ha elaborando reuniendo y clasificando algunos aspectos significativos de los relatos leídos, con ello pretendemos plasmar los saberes que orientan su praxis. La ubicación de estas citas corresponde a un modo de ordenar, no de modificar, el contenido de las mismas.

\section{Lenguaje}

"Sé... donde tengo que decir las cosas, o... no sé, deben haber todas estas palabras para explicarse uno, para poder seguir diciendo más cosas" (Margarita).

\section{Felicidad}

"El ver a mis hijos felices, eso más que nada, ver a mis hijos bien" (Rosa).

\section{Instituciones}

"No me van a escuchar" (María).

\section{Dinero}

"El campesino está... siempre, anda siempre sin plata, esté con plata o sin plata hace el trabajo igual no más, no le toma importancia que tenga que tener plata para hace esto, él trabaja sin plata no más, porque él sabe que lo que está haciendo algún día le va a dar fruto, por eso el campesino trabaja con la esperanza que en el día de mañana va a tener, el campesino sabe esperar, ahí está la diferencia" (Manuel).

\section{Pobreza}

"Porque un pobre no puede llegar a ser lo que quiere ser" (Ubelinda).

\section{Educación}

"Yo misma, imagínese, falta de educación poh, a veces uno se quisiera explicar, no se ahí... ahi se queda, no tiene palabras... se quisiera explicar, pero no tiene" (Rosa). 


\section{Vida}

"Yo realmente me he educado solo, por lo que he andado, por lo que he vivido, porque yo he caminado mucho" (Manuel).

\section{Trabajo}

"Aquí uno trabaja a la voluntad de uno no más, nadie lo manda mucho, que como en otros lados, como en la ciudad" (Juan).

\section{Emigración}

"Muchos que no les gusta el campo, mucha gente que no le gusta el campo así que no quieren estar, prefieren no embarrarse y mejor irse a trabajar a la ciudad" (Juan).

\section{Formas de ser}

"Así la persona del pueblo es más dura, si como más dura, no es igual que la persona del campo, la persona del campo es como más, más sensible" (Aurora).

\section{Subsistencia}

"Porque en el campo con lo que uno siembra se asegura el pan para el año... el pan para el año y tiene uno alimento para su aves... entonces todas esas cosas uno se salva, porque tiene comida... aunque no tiene plata, pero tiene comida" (Roberto).

\section{Cambios}

"Antes si poh, porque yo mismo lo que he vivido... uno ha vivido más como se dice en la gloria, porque lo ha tenido todo, me entiende, ha tenido todo, mis papás... lo han dado de todo a nosotros, y antes por lo que me cuenta mi papá... lo que no, lo que yo tengo ahora, ellos antes no lo tenían, porque antes era muy sacrificada la vida en el campo, antes... ellos tenían que trabajar de sol a sol para poder... llegar con el pago mensual a la casa" (Adrián).

\section{Costumbres campesinas}

"Eran de esas bolsas de algodón que antes había, que salían en la harina, en la harina salía antes y eso se usaba mucho en el campo, nos hacían ropa a nosotros, a los niños se les hacía ropa, a mi me hacían camisitas para que me pusiera debajo" (María).

\section{Libertad}

"Aquí hay más libertad para ir donde uno quiera" (Juan).

\section{Familia}

"De ayudarle al papá, de encargarse de los animalitos, eso. Que tiene que hacer porque es su deber pa' que sea una familia unida" (Rosa).

\section{La ciudad}

"La desventaja es que están casi muy encerrado" (Ubelinda).

\section{El Campo}

"Siempre me ha gustado, por eso estoy aquí, se hace difícil un poco, pero hay que echarle adelante no más... lo que se pueda" (Manuel).

\section{Sacrificio}

"Lo que es en trabajo, el trabajo es demasiado sacrificado para toda persona, y uno no quiere eso para los hijos, es lindo vivir en el campo, pero lo que es el trabajo no... demasiado sacrificado, uno tiene que estar ocho horas parada a todo sol, o al viento, la lluvia, al frío" (Ubelinda).

\section{Amistad}

"Si porque de todas maneras uno debe tener amigos, sea donde sea es importante, porque o sino sería como vivir en el desierto" (Roberto).

\section{Equidad de género}

"Ya en este momento a nosotras nos mandan a hacer todos los trabajos, el trabajo que sea que lo pueda hacer un hombre, nosotras igual somos capaces de hacerlo igual que ellos, pero la paga no es igual" (Rosa).

\section{Temores}

"De que así como está el tiempo, como vienen los días, que no pueda... que pueda haber una guerra, o que los sembrados no den por mucha sequía, a veces se secan así sin dar, y yo digo que más adelante a lo mejor ya no vamos a cosechar. $Y$ en el invierno igual, las Iluvias que se aproximan. Eso son mis temores" (Teresa).

\section{Paciencia}

"Saber esperar, así como uno bota la semilla en la tierra, uno tiene que saber esperar y por qué no voy a esperar de mis hijos" (Diego).

\section{CONCLUSIONES}

Estas citas dan cuenta de los sistemas cognitivos que construyen estos/as informantes de sectores rurales del centro y sur de Chile, expresadas por medio del lenguaje. Ellas muestran el apego a un lugar, los recuerdos, la valoración de la familia, su visión del mundo, y se traducen en un extenso y complejo sistema de creencias que orientan su proyecto de vida.

Estas citas muestran saberes que responden a una situación, una época y un territorio particular, así como a proyectos específicos de una situación social singular. Sus representaciones expresan saberes que son herencia de las generaciones pasadas, expresan saberes emergentes, saberes sustituidos y saberes híbridos (Núñez, 2004). Son saberes que poseen metáforas propias, cuyo referente principal es la naturaleza. 
Estas citas, son sólo una pequeña muestra del inmenso conocimiento acumulado generación tras generación, mismo que sin embargo sigue en continua construcción, adaptando formas a medida que la realidad se las impone. Aún así, consideramos que son suficientes para avanzar en un diálogo que rechace la violencia de la homogenización y se enfrente a prácticas que buscan la sumisión de voluntades y visiones que se expresan en forma distinta al discurso universal sobre lo que es la vida o como vivirla. Estas citas nos ayudan además, a tener más elementos para reconocer las culturas olvidadas por la modernidad y las globalizaciones.

Se re-conoce y traduce la relación que tienen con los/as otros/as, la supremacía de la paciencia, del "andar"; por otra parte, las categorías de tiempo y espacio en la forma de vivir su vida son una muestra de que hay otras formas de conocer, entender y por tanto de construir la realidad.

Destaca que para ellos/as, el saber consiste precisamente en abordarlo con otros/as y pocas veces desde el individualismo. Sin embargo, se observa en el diálogo con estos/as interlocutores/as, que han perdido la memoria y la palabra, pues sus saberes tradicionales han sido sepultados por la modernidad impuesta. Ellos parecen no haber podido escapar a la lengua dominante. Sus aspiraciones, problemas y anhelos son conceptualizados desde el discurso occidental y desde el paraguas de los valores universales, elaborados por una raza, por un género y por una clase social determinada.
Así, el primer paso para recuperar la memoria sería convertir el saber en indagación, en elucidación y esclarecimiento de palabras borradas. Para ello podría servir profundizar en la propuesta de De Santos de Sousa (2003) adentrándonos en una sociología del silencio y en una teoría de la traducción para hacer conmensurables los distintos saberes.

Finalmente, esperamos haber actuado sin violencia simbólica, sin haber aplicado la fuerza a los saberes construidos por seres que muchas veces han sido subyugados/as, marcados por nuestra certeza que eran "ignorantes" o donde su "sabiduría" sólo ha sido tratada como un mero ejercicio "romántico". Quisiéramos entonces, haber utilizado la máxima consideración a la hora de hacer públicas estas reflexiones y hallazgos.

En conclusión, los saberes que constituyen estos breves enunciados conforman una constelación de sentidos que organizan prácticas culturales y productivas. Es un saber que no renuncia a la razón, pero que la irriga con sensibilidades, sentimientos y sentidos. Esperamos haber logrado establecer una relación de otredad entre seres diferenciados/as: ustedes los/as lectores/as, ellos/as los/as campesinos/as y nosotras las autoras.

\section{AGRADECIMIENTOS}

Agradecemos los aportes realizados por Elda Millan Ghisleri.
1. FAO y UNESCO (Estudio conjunto). Educación para el desarrollo rural: hacia nuevas respuestas de política [en línea]. s/f. [citado 08 mayo 2009; 16,30 EST]. Disponible en World Wide Web: http:// www.fao.org/SD/ERP/0-educacion\%20 rural\%20ext.pdf

2. Op.cit.

3. Departamento de asuntos económicos y sociales. División de desarrollo sostenible: Programa 21 [en línea]. s/f. [citado 16 mayo 2009; 20:00 EST]. Disponible en World Wide Web: http:// www.un.org/spanish/esa/sustdev/ agenda21/index.htm
4. UNESCO. Convención Para la salvaguardia del patrimonio cultural inmaterial [en línea]. París, 17 de octubre 2003. [citado 24 de abril 2009; 09:50 EST]. p.3. Disponible en World Wide Web: http://unesdoc.unesco.org/ images/0013/001325/132540s.pdf

5. UNESCO. Patrimonio cultural inmaterial [en línea]. 23 de marzo 2009. [citado 02 de Junio 2009; 11:30 EST]. Disponible en World Wide Web: http://www.unesco. org/culture/ich/index.php?pg=00052

6. UNESCO. Cultura [en línea]. s/f. [citado 28 de mayo 2009; 09:00 EST]. Disponible en World Wide Web: http://portal.
unesco.org/culture/es/ev.php-URL $I D=29916 \& U R L$ DO=DO_TOPIC\&URL_ SECTION=201.html

7. GRAIN. Biodiversidad: La agricultura, sus saberes y cuidados [en línea]. Enero de 2009. [citado 02 mayo 2009; 15:30 EST]. Disponible en World Wide Web: http:// www.grain.org/biodiversidad/?id $=421$ 
Bauman, Z. (2003). Modernidad líquida. México: Fondo de Cultura Económica.

Bauman, Z. (2005). Los restos de la educación en la modernidad líquida. Barcelona: Gedisa.

Castells, M.; Giddens, A. y Touraine, A (2002). Teorías para una nueva sociedad. España: Fundación Marcelino Botín.

Cubero, R. (2005). Perspectivas constructivistas. Barcelona: Graó.

De Sousa Santos, B. (2003). Crítica de la razón indolente. Contra el desperdicio de la experiencia. Bilbao: Desclée.

Fals Borda, O. (1973). Ciencia propia y coIonialismo intelectual. México: Nuestro Tiempo.
Flick, U. (2004). Introducción a la investigación cualitativa. Madrid: Morata.

Fourez, G. (2008). Cómo se elabora el conocimiento -la epistemología desde un enfoque socioconstructivista. Madrid: Narcea.

Freire, P. (1998). ¿Extensión o comunicación? La concientización en el medio rural. México: Siglo XXI.

Gil, F. y Jover, G. (2000). Las tendencias narrativas en pedagogía y la aproximación biográfica al mundo infantil. Revista Enrahonar, № 31, 107-122.

Leff, E. (2002). La transición hacia el desarrollo sustentable: perspectivas de América Latina y el Caribe. México: Instituto Nacional de Ecología.
Morín, E. (1988). El método: El conocimiento del conocimiento. Madrid: Cátedra.

Morín, E. (1990). Introducción al pensamiento complejo. Barcelona: Gedisa.

Núñez, J. (2004). Los saberes campesinos: Implicaciones para una educación rural. Revista Investigación y Postgrado, Vol.19, №2, 13-60.

Ulrich, B. (1998). ¿Qué es la globalización? Falacias del globalismo, respuestas a la globalización. Barcelona: Paidos. 